\title{
THE VORTEX-BOUNDARY ELEMENT METHOD: NEW PRESSURE METHODS FOR APPLICATION TO THE EXTERNAL FLOW NOISE PROBLEM
}

\author{
Laurine Leep-Apolloni* and Gary S. Strumolo ${ }^{\dagger}$ \\ Ford Motor Co., Dearborn, MI \\ David Dowling ${ }^{\ddagger}$ \\ University of Michigan, Ann Arbor, MI
}

\begin{abstract}
Three new methods to compute pressure within a vortex method are developed and presented. Although demonstrated in a Vortex-Boundary Element Method, these pressure methods are suitable for any vortex method. They have computational savings of 2-3 orders of magnitude over a standard Poisson pressure method when surface pressure at a small set of locations is desired. This savings is significant as the computational time needed to solve for the pressure field using a Poisson method exceeds that of the VBEM for cases similar to those studied here. These methods are validated for flow on the front face of a cube set orthogonal to a uniform freestream flow. Flow about a cube yawed at $30^{\circ}$ to the streamwise direction is simulated. Timeaveraged flow field data and surface pressure spectra are presented for Reynolds numbers of 500 and 1500 . The results of the calculations. correctly predict an overall sound pressure level increase with increasing Reynolds numbers. The VBEM also captures the different levels of pressure fluctuations associated with different flow regions. This work also serves as a successful first step in demonstrating the potential of the VBEM for use in computing unsteady flows to predict the pressure spectrum associated with the unsteady surface pressure fluctuations.
\end{abstract}

\section{INTRODUCTION}

The increased capabilities of computers both in terms of memory and speed have caused fluid mechanics researchers to move from simulating

\footnotetext{
${ }^{*}$ Technical Specialist

${ }^{\dagger}$ Senior Staff Technical Specialist

‡ Assistant Professor

Copyright $(1999$ by Ford Motor Co. Published by the American Institute of Aeronautics and

Astronautics, Inc.; with permission.
}

steady state flows to modeling fully transient flow phenomena. Accurate time-dependent solutions give hope that industrial acoustic problems, such as automotive aerodynamic noise, can be addressed directly rather than relying on approximate analytical and phenomenological methods.

Automobile aerodynamics noise is defined as the noise generated by the flow of air around the exterior of the car. This noise is of concern to those both inside and outside of the vehicle. The unsteady flow around the car exterior can cause sound internal to the vehicle by vibrating its panels and windows which then radiate sound to the vehicle interior. The exterior flow can cause noise which is propagated away from the automobile and heard by observers outside the vehicle. Of primary concern, especially in the U.S., is the interior sound caused by wind noise.

Because traditional CFD methods such as finite difference RANS codes with turbulence closure models have not proven successful in computing the time-accurate external flow about an automobile for the purpose of predicting wind noise, alternative methods are being explored. Vortex methods are attractive because they only requires a surface mesh (rather than a complete volume mesh) and have, in some cases, reproduced unsteady vortex shedding for flow over a channel ${ }^{1,2}$, highlighting its potential to predict the three-dimensional unsteady shedding of interest in the automotive applications. In order to predict aerodynamic noise, the problem of computing the surface pressure must be addressed. Since vortex methods work in terms of velocity and vorticity, the pressure must be determined through additional equation solving.

The vortex method used in this work to compute the unsteady flow field is the Vortex-Boundary Element Method (VBEM) developed at MIT by A.F. Ghoniem and his colleagues ${ }^{1,2,3,4}$. The pressure methods implemented in this code, however, are applicable to any vortex technique. 
The most obvious way to obtain surface pressures from a vortex code is to solve the Poisson pressure equations throughout the flow field. Since a Poisson solver requires the use of a grid which fills the cntire flow domain, it negates the benefits and beauty of the vortex method that uses only a surface mosh. Also, a vast amount of unnecessary work is done to compute the pressure throughout the whole domain when it is only needed at specific points on the surface. While the Poisson method may be palatable when only steady state results are of interest, thereby requiring the Poisson equations to be solved only once, computations for the purpose of predicting the surface pressure spectra necessitate computing the pressure at each time step.

Three methods to obtain surface pressure are examined in this paper:, the Lagrangian method, the Eulerian method, and the vorticity flux method. The Fulerian method involves the insertion of small computational chimneys into the domain which connect the surface location where the pressure is desired to the freestream or a point where pressure is know. The Lagrangian method computes the surface pressure by using a set of tracer particles which are released along a line connecting the point where pressure is desired to a point where the pressure is known, such as the freestream. These tracer particles are released at every time step an tracked for a single time step. Both of these methods are broadly applicable and can compute the pressure at arbitrary locations, including the body surface. The Eulerian method was found to be the more robust method. A computational saving of two to three orders of magnitude over a Poisson solver is seen when one of the newly developed methods is used to compute the pressure at a small number of locations. The vorticity flux method computes the pressure only at surface locations by integrating the vorticity generated at the surface from a point where the pressure is assumed to be known, such as a stagnation point, to the surface location of interest. This method was found to be unsuitable for the particular application of interest due to its inability to integrate the surface pressure past points of flow separation, but is applicable for more streamlined geometries where there are no separation points.

The present work uses flow around a cube as the test problem. Although the body is a very simple shape, three important flow regimes can be studied by simulating the flow around a cube yawed 30 degrees: separated, attached, and transitional. The vortex shedding on the top and bottom of the yawed box is very similar to the vortex shedding which creates wind noisc on automobiles.

\section{NUMERICAL METHODOLOGY}

Because the VBEM utilizes velocity and vorticity as its prime variables, it is necessary to develop a complimentary method to determine pressure. In the particular application of interest, only surface pressure at specific locations is desired; however, these surface pressures must be calculated at the conclusion of each step. The methods utilized in this work were chosen to be the most efficient for the intended purpose while other, more computationally intensive, methods for computing the entire pressure field are possible (Poisson solver and boundary integral method ${ }^{5}$ ), they were not explored. The methods for calculating time accurate surface pressure investigated are: the vorticity flux method, the Eulerian method, and the Lagrangian method.

\section{Vorticity Flux Method}

A method which arises as an extension of a twodimensional vortex method developed by Lewis ${ }^{6}$ is to use the surface vorticity flux at each time step to determine the surface pressure gradient at any or all surface locations. These gradients can then be used to obtain absolute pressure values by integrating along a path from a known pressure (e.g., a stagnation point) to any other point on the surface. The complete vorticity flux method derivation is shown for a two-dimensional case by Lewis ${ }^{6}$.

Lewis' method equates the surface vorticity production to the surface pressure gradient as

$$
\frac{d \gamma(s)}{d t}=-\frac{1}{\rho} \frac{d p}{d s}
$$

for a two-dimensional case where $s$ denotes the direction tangent to the surface. The surface vorticity is equal to the slip velocity outside the boundary layer,

$$
\gamma(s)=v_{s}
$$

so Eq. (1) can be written as

$$
\frac{d v_{s}(s)}{d t}=-\frac{1}{\rho} \frac{d p}{d s} .
$$

This equation is basically a statement of the Euler Equation, the momentum equation with the viscous diffusion is neglected. The acceleration of the fluid, $d v_{s} / d t$, can be computed via a spatial differencing of the square of velocity

$$
\frac{d v(s)}{d t}=\frac{d}{d s}\left(\frac{v_{s}{ }^{2}}{2}\right) \text {. }
$$

Substituting (4) into (3) results in a new expression for surface pressure gradient 


$$
\frac{d p}{d s}=-\rho \frac{d}{d s}\left(\frac{v_{s}{ }^{2}}{2}\right) .
$$

This equation can be converted into a threedimensional form; thus the surface pressure derivative in the $\mathrm{x}$-direction is

$$
\frac{\partial p}{\partial x}=-\rho \frac{\partial}{\partial x}\left(\frac{u_{s}^{2}}{2}\right) \text {. }
$$

The pressure gradients are easily calculated from the slip velocities which are already available within the current code. To compute the surface pressure as a specific location, only integration of the pressure gradient along a path from a surface location of constant pressure to the desired surface location is nccessary.

\section{Eulerian Method}

The Eulerian method involves the insertion of small computational chimneys into the domain which connect the surface location where the pressure is desired to the freestream or a point where the pressure is known. This method is named the Eulerian method because the calculations of the surface pressure are based on differencing the Navier-Stokes equations over a fixed grid through which the fluid flows. To implement this method, a small $5 \times P$ grid stencil column is constructed which connects the body surface, at the location where the surface pressure is desired, to the freestream as shown in Fig. 1. The number of grid points in the ydirection, $\mathrm{P}$, is chosen to adequately resolve the flow field.

This methods starts with the Eulerian form of the Navier-Stokes equations

$$
\frac{D \vec{u}}{D t}=\frac{\partial \vec{u}}{\partial t}+\vec{u} \cdot \nabla \vec{u}=-\frac{1}{\rho} \nabla p+\nabla^{2} \vec{u}
$$

the y-component of which is

$$
\frac{\partial p}{\partial y}=\mu \nabla^{2} v-\rho\left(\frac{\partial v}{\partial t}+\tilde{u} \cdot \nabla v\right)
$$

The pressure at the surface of the object can be obtained by integrating Eq. (8) along a line from a known pressure, $p_{\infty}$, to a point $s$ on the surface

$$
\begin{aligned}
& p_{\infty}-p_{s}=\mu \int_{i}\left(\frac{\partial^{2} v}{\partial x^{2}}+\frac{\partial^{2} v}{\partial y^{2}}+\frac{\partial^{2} v}{\partial z^{2}}\right) d y-\rho \int_{i} \frac{\partial}{\partial} d y \\
& -\rho \int_{i}\left(u \frac{\partial}{\partial x}+v \frac{\partial}{\partial y}+w \frac{\partial v}{\partial z}\right) d y
\end{aligned}
$$

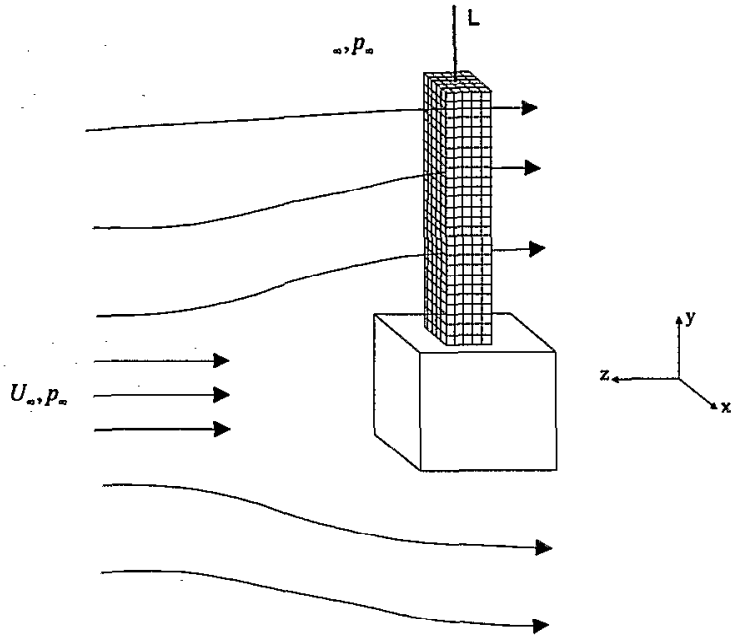

Figure 1. Eulerian method schematic.

This method introduces numerical error through the calculations of the first and second derivatives of the velocity. It is also somewhat tedious as these quantities must be determined at each of the grid points and at each time step. This method is somewhat similar to the most obvious method for computing the pressure field which involves solving the Poisson pressure equation at grid points which are distributed throughout the flow field by inverting a very large matrix or using an iterative Poisson solver. The three-dimensional Poisson equation which would be solved is

$$
\nabla^{2} p=2 \rho\left\{\begin{array}{l}
\left(\frac{\partial u}{\partial x} \frac{\partial v}{\partial y}-\frac{\partial u}{\partial y} \frac{\partial v}{\partial x}\right)+ \\
\left(\frac{\partial u}{\partial x} \frac{\partial w}{\partial z}-\frac{\partial u}{\partial z} \frac{\partial w}{\partial x}\right)+ \\
\left(\frac{\partial v}{\partial y} \frac{\partial w}{\partial z}-\frac{\partial v}{\partial z} \frac{\partial w}{\partial y}\right)
\end{array}\right\}
$$

The Poisson method is similar to the Eulerian method in that they both involve the computation of velocity gradients at fixed grid locations; the Eulerian method used herein, however, is not as computationally intensive as solving the Poisson Equation for the entire domain at each time step.

\section{Lagrangian Method}

In this method the viscous portion of the NavierStokes equations is ignored and the Lagrangian version of the Euler Equation

$$
\rho \frac{D \vec{u}}{D t}=-\nabla p
$$

is exploited. Although this method uses the Euler Equation, it is called the Lagrangian method because 
it uses a set a particles which are tracked in a Lagrangian fashion to compute the pressure. A diagram of this method is shown below in Fig. 2 .

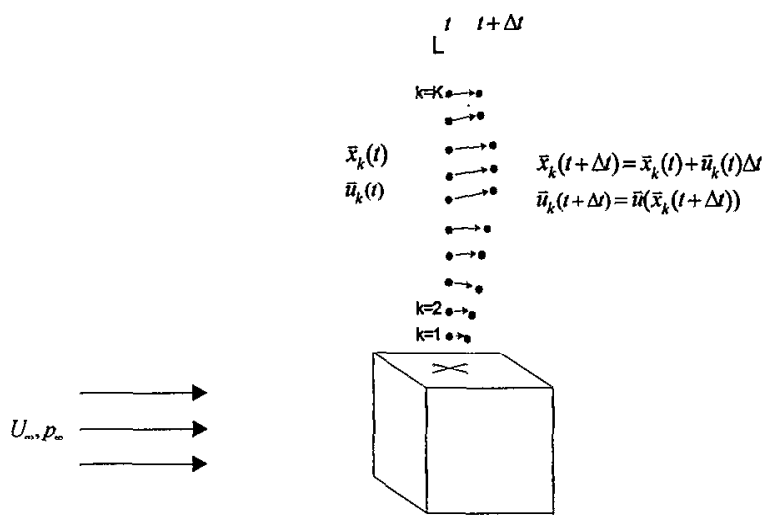

Figure 2. Lagrangian method schematic.

The method works as follows:

1. At time t, a set of particles, each at a given location $\bar{x}_{k_{0}}$, are released into the flow which initially lie along a line $\mathrm{L}$, say a line in the $y$ direction. The velocity at each point is $\vec{u}_{k_{0}}=\vec{u}_{k}\left(\vec{x}_{k_{0}}, t\right)$.

2. These particles are then moved over Dt, according to their particle velocities at their original locations:

$$
\vec{x}_{k}(t+\Delta t)=\vec{x}_{k_{0}}(t)+\vec{u}_{k}\left(\vec{x}_{k_{0}}, t\right) \Delta t .
$$

3. The velocity of the flow field at the new location of the particle is found

$$
\bar{u}_{k}(t+\Delta t)=\vec{u}\left(\vec{x}_{k}(t+\Delta t)\right) \text {. }
$$

4. The acceleration of the particle is then approximated using

$$
\frac{D \vec{u}}{D t} \approx \frac{\vec{u}_{k}-\vec{u}_{k_{0}}}{\Delta t} .
$$

5. The pressure gradient in the y-direction can be approximated at the locations along the line L, by the y-component of Eq.(14)

$$
\frac{\partial p}{\partial y}=-\rho \frac{D v}{D t} \approx-\rho \frac{v_{k}-v_{k_{0}}}{\Delta t}
$$

6. The surface pressure can be found by integrating Eq. (15) from $p_{\infty}$ according to

$$
p_{\text {surace }}=p_{\infty}+\sum_{k=1}^{K} \rho \frac{v_{k}-v_{k_{0}}}{\Delta t} \Delta y \text {. }
$$

This process is repeated at each time step, with new particles being released at the initial points along line $L$ and allowed to move position over 1 time step (the particles are not tracked after that). Since the movement of the particles is only tracked for one time step, the error associated with neglecting the diffusion term should be small.

\section{METHOD VALIDATION}

In order to validate the pressure schemes, the average surface pressure was computed by means of all three pressure methods at locations on the front face of the cube for the zero yaw cube and a Reynolds number of 500 . The time-varying probe pressures were recorded and subsequently averaged to provide mean values. The time-averaged pressures are expressed in terms of pressure coefficient, $\mathrm{Cp}$.

Pressure coefficients as computed by the three methods for a vertical traverse (y-direction) and horizontal traverse (x-direction) from the center of the face to the top of the box are shown in Fig. 3. For comparison, surface pressure coefficient data as computed by the commercial lattice gas code, PowerFlow, are shown as solid circles. PowerFlow calculates pressure in addition to velocity as part of the flow solution. It is assumed that both the VBEM and PowerFlow correctly predict the unseparated flow field on the front of the cube and that these comparisons check the pressure computed by the newly implemented pressure methods in the VBEM.

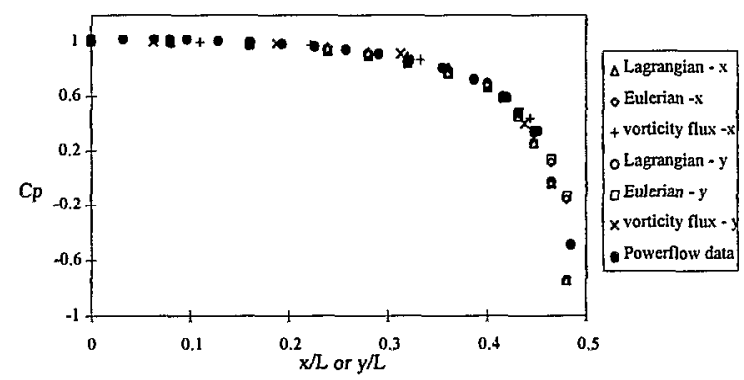

Figure 3. Pressure coefficient on the front face of a cube yawed at zero degrees to the streamwise direction at $\mathrm{Re}=500$.

The pressures in the Eulerian and Lagrangian methods are computed with respect to the freestream pressure whereas the pressure in the vorticity flux method is computed relative to a known pressure on the surface; in this case, it is computed relative to the stagnation pressure which is assumed to be constant and located at the geometric center of the face. Hence the $\mathrm{Cp}$ at the center of the front face is set to unity in the vorticity flux method but calculated absolutely in the Eulerian and Lagrangian methods.

The results of the Eulerian method, shown for two traverses from the center of the face, agree well 
with the PowerFlow data at all locations. The Lagrangian method shows a small amount of error toward the outside of the face where the pressure gradient is high. The underprediction of the pressure coefficient in the Lagrangian method is an artifact of the forward differencing of the velocity used to estimate the acceleration of the fluid at points along the line connecting the body to the freestream. The size of the error is a function of the magnitude of the velocity component tangent to the surface at the computational probe location and the size time step used. As the time step is decreased, the error always decreases. The error increases in areas where the velocity tangent to the surface increases. Thus near the center of the face where the velocity is normal to the face, decreasing from the freestream value to zero, the error is very small for a wide range of time steps. At position at greater radial distance from the face center where the velocity tangent to the facc has increased, the errors increase. In this particular flow situation, the Lagrangian method predicts a pressure value at a location slightly further from the center than the computational probe. In Fig. 3, the error in the Lagrangian method is small but yet perceivable. Thus when using the Lagrangian method it is imperative that a small time step be used. For computations used to capture the sound spectrum the time step is sufficiently small for the two methods to yield nearly identical pressures.

The four points shown for the vorticity flux method are computed at the surface tile centers (there are eight tiles in the $y$-direction and nine tiles in the $x$-direction with each traverse only crossing half of the tiles). The vorticity flux method results show good agreement with the other two methods and the PowerFlow data within the discretization limitations. Despite the favorable results shown for the pressure on the front of the cube, the vorticity flux method is not able to compute the pressure on the top of the cube because of the jump in pressure at the sharp corner of the box which is along the integration path. Other abrupt changes in the pressure due to flow structure (separation/reattachment) would also be problematic for the vorticity flux method. Due to these limitations and hence inapplicability to the problem at hand, no further work was conducted using the vorticity flux method in this investigation.

The pressure coefficient distribution on the front face of the cube computed by the Lagrangian and Eulerian methods agrees well with data computed by another computational method. Furthermore, the results show agreement with experimental data from similar geometric configurations. Thus it can be concluded that the pressure methods are capable of computing the pressure at arbitrary points in the flow from the available VBEM flow field information.

\section{COMPUTATIONAL RESOURCE COMPARISON}

The Lagrangian and Eulerian methods differ in computational resource demands, both in CPU usage and memory requirement. The Eulerian method requires both more memory and more CPU time. The memory requirements per probe are twice that of the Lagrangian method; however, as a portion of total memory required for a simulation similar to those computed in this work, the difference between the methods is quite small. The most significant difference between the two methods is the number of points at which the velocity must be computed, which requires the potential and vortical portions of velocity to be computed for an arbitrary spatial location. In the case of the Eulerian method, a fixed $5 \times P$ stencil is used ( $P$ is the number of grid points between the probe location on the body and the freestream), requiring the velocity to be computed $5 \mathrm{P}$ times for each probe at each time step. The Lagrangian method uses a two-step method, which requires the calculation of velocities at $2 P$ locations at each time step. The other computations associated with each of the methods are small relative to the computation of the velocities, hence the overall number of floating point operations required for the Eulerian method is over twice that for the Lagrangian method. Thus, if the time step used for the calculation is quite small, the Lagrangian method is recommended since it requires less resources than the Eulerian method.

A rough analysis was made of the total floating point operations required for the Lagrangian and Eulerian pressure method as compared to an estimate of the number of operations needed to compute the entire pressure field using a direct Poisson solver. The operation count for the Poisson solver comes from work by Babu and Korpela ${ }^{7}$. The parameters used in this analysis are:

$$
\begin{aligned}
& \mathrm{N}= \text { number of vortices in the domain } \\
& \mathrm{M}= \text { number of boundary elements on surfaces } \\
& \mathrm{P}= \text { number of points connecting the surface } \\
& \text { to freestream in Eulerian and Lagrangian } \\
& \text { methods } \\
& \mathrm{I}, \mathrm{J}, \mathrm{K}= \text { number of } \mathrm{x}-, \mathrm{y}-\text {, and } \mathrm{z} \text {-direction grid } \\
& \text { points for Poisson method }
\end{aligned}
$$

The number of operations for the basic VBEM (not computing pressure) is $O\left(\mathrm{~N}^{2}+\mathrm{NM}\right)$. The operation count for the Eulerian and Lagrangian 
pressure methods as compared to the Poisson method ase:

$$
\begin{gathered}
\mathrm{n}_{\mathrm{Poisson}}=(\mathrm{N}+\mathrm{M}+20)^{*} \mathrm{I}^{*} \mathrm{~J} * \mathrm{~K}+2 *\left(\mathrm{I}^{*} \mathrm{~J}^{*} \mathrm{~K}^{2}+\mathrm{I}^{*} \mathrm{~K}^{*} \mathrm{~J}^{2}\right) \\
\mathrm{n}_{\text {Eulerian }}=5^{*} \mathrm{P}(\mathrm{N}+\mathrm{M})+34 \mathrm{P} \sim 5^{*} \mathrm{P} *(\mathrm{~N}+\mathrm{M}) \\
\mathrm{n}_{\text {Lagrangian }}=2^{*} \mathrm{P} *(\mathrm{~N}+\mathrm{M})+13 \mathrm{P} \sim 2^{*} \mathrm{P} *(\mathrm{~N}+\mathrm{M})
\end{gathered}
$$

Operation count estimates for the three methods are compared in Table 1 for a range of parameters. These estimates show that for a typical case where there are 10,000 vortex elements in the system, 700 boundary elements, 200 points are used to connect the freestream and the body, and a $100 \times 100 \times 100$ grid is used for the Poisson solver, only $0.04 \%$ of the operations necessary to compute the entire pressure filed are needed to compute the pressure at a single probe locations using the Lagrangian method (or
$0.1 \%$ for the Eulerian method). In other words the pressure at 2500 locations could be computed in the same number of operations as using the Poisson method. Thus, in applications where the pressure at only a few locations is desired, either of the proposed methods would reduce the computational requirement by 2-3 orders of magnitude.

The savings gained by using one of the new pressure methods is of great significance since the computational time necessary to solve the pressure field using a Poisson Method exceeds that of the VBEM when the number of grid points on which the pressure is solved exceeds the number of vortex elements. For example, a $22 \times 22 \times 22$ mesh has grid points in excess of 10,000 and a $28 \times 28 \times 28$ mesh has over 20,000 grid points. Thus, for cases similar to those in this work, the computational time of the Poisson method would far exceed that of VBEM.

Table 1. Floating point operation count comparison

\begin{tabular}{cccc||ccc||ccc}
\hline$N$ & $M$ & $P$ & $I, J, K$ & Eulerian & Lagrangian & Poisson & $E / L$ & $P / E$ & $P / L$ \\
\hline 10000 & 700 & 200 & 50 & $1.07 \mathrm{E}+07$ & $4.28 \mathrm{E}+06$ & $1.35 \mathrm{E}+09$ & 2.5 & 126 & 316 \\
10000 & 700 & 200 & 75 & $1.07 \mathrm{E}+07$ & $4.28 \mathrm{E}+06$ & $4.59 \mathrm{E}+09$ & 2.5 & 428 & 1071 \\
\hline 10000 & 700 & 200 & 100 & $1.07 \mathrm{E}+07$ & $428 \mathrm{E}+06$ & $1.09 \mathrm{E}+10$ & 2.5 & 1020 & 2550 \\
10000 & 700 & 200 & 150 & $1.07 \mathrm{E}+07$ & $4.28 \mathrm{E}+06$ & $3.72 \mathrm{E}+10$ & 2.5 & 3474 & 8685 \\
10000 & 700 & 100 & 50 & $5.35 \mathrm{E}+06$ & $2.14 \mathrm{E}+06$ & $1.35 \mathrm{E}+09$ & 2.5 & 253 & 632 \\
10000 & 700 & 300 & 50 & $1.61 \mathrm{E}+07$ & $6.42 \mathrm{E}+06$ & $1.35 \mathrm{E}+09$ & 2.5 & 84 & 211 \\
20000 & 700 & 200 & 100 & $2.07 \mathrm{E}+07$ & $8.28 \mathrm{E}+06$ & $2.09 \mathrm{E}+10$ & 2.5 & 1010 & 2526 \\
\hline
\end{tabular}

\section{RESULTS}

The geometry studied is a free-floating cube located in the center of a large channel of square cross section. The channel or tunnel dimensions are $16 \times 16 \times 10$ in non-dimensional units, where a cube side has the dimension of unity. The cube is located along the tunnel centerline with three cube lengths upstream of the cube and six lengths downstream of the cube. Each tunnel wall is divided into 16 elements and each cube side into 100 elements for use in the boundary element portion of the computations. The tiles which produce the vortices carpet only the sides of the cube, with the tiling set at $9 \times 8 \times 8$ (i.e., $9 \times 8$ on the $x-y$ sides, $8 \times 8$ on the $y-z$ sides, $9 \times 8$ on the $x-z$ sides) for all the simulations. The geometry configuration considered is the cube yawed at $30^{\circ}$ to the streamwise direction. The simulations were run at Reynolds numbers of 500 and 1500. The flow was impulsively started at $t=0$, using a uniform nondimensional velocity $\mathrm{U}=1.0$ throughout the domain. The inlet velocity was maintained at $U_{\mathrm{in}}=1.0$ for the entire simulation.

With the flow started impulsively at time $\mathrm{t}=0 \mathrm{a}$ start-up period is initially observed, followed by a fully developed state. A fully developed or steady state is reached when the number of vortex elements resident in the domain remains nearly constant form time step to time step, the number of new vortex elements generated balancing the number exiting the domain. Although the fully developed flow is transient in nature, these computed flow fields are most compactly viewed in terms of time-averaged results.

Time-averaged flow results are shown in Figs. 4 and 5 for Reynolds numbers of 500 and 1500, respectively. These figures display horizontal slices through the flow ficld just above the top cubc surface. In these cases, one might expect that two vortices would be shed off the front two edges of the 
cube as indicated by work of Stapleford and Carr ${ }^{8}$ and Hucho'. However, since these comparative experimental studies were performed at much higher Reynolds numbers, it is unclear what type of flow might develop in the lower Reynolds number regime.

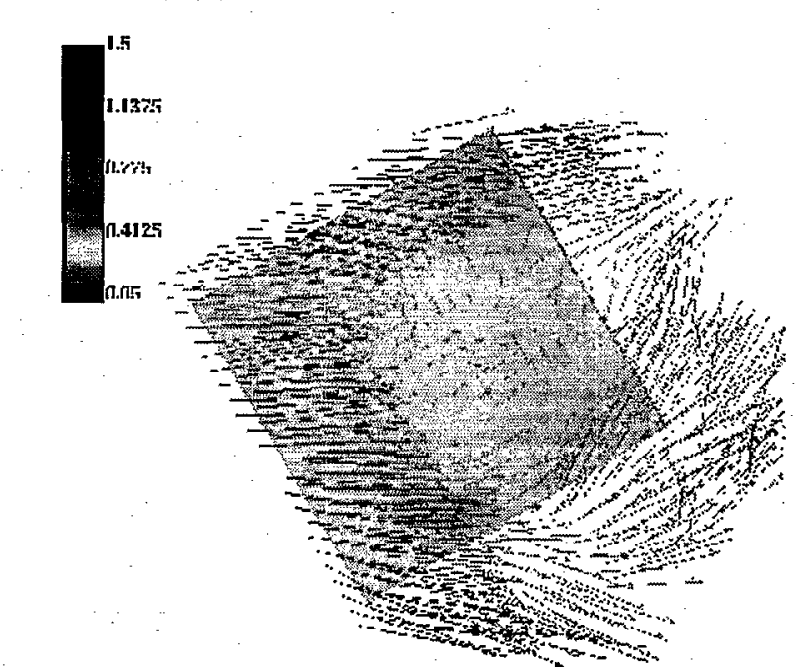

Figure 4. Time-averaged flow results for flow past a yawed cube at $R e=500$. Horizontal slice is just above the top surface.

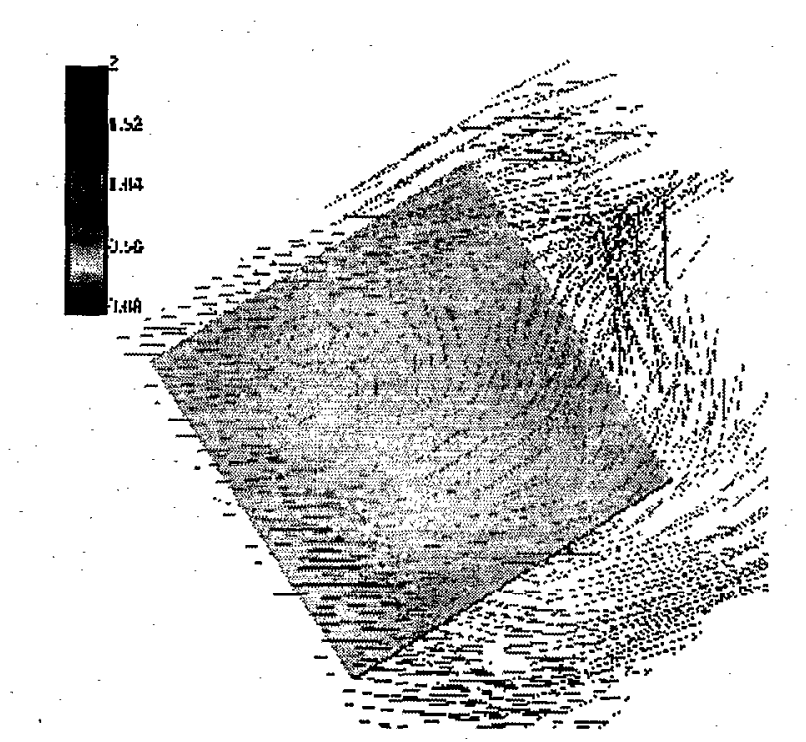

Figure 5. Time-averaged flow results for flow past a yawed cube at $R e=1500$. Horizontal slice is just above the top surface.

In both the cases simulated, a recirculation zone develops behind the leading corner of the cube. The flow turns around these recirculation zone, but true vortices are not shed from the leading edges. One can observe the flow features becoming stronger with increased Reynolds number. In Fig. 5 the recirculation zone has moved upstream on the cube causing the flow to turn more sharply. Further increase in Reynolds number would likely show separation occurring. Simulations were not performed at higher Reynolds numbers due to computational limitations.

Pressure-time data for seven probe locations were gathered at each Reynolds number. The probe locations are shown in Fig. 6. The time step for these simulations was chosen based on Nyquist sampling theory in order to capture frequencies up to $1000 \mathrm{~Hz}$. The simulations were fun for at least 10 periods of the lowest desired frequency, $50 \mathrm{~Hz}$. The data was analyzed using a power spectrum analysis method. The output of this method, $\mathrm{p}_{\text {rms }}^{2}$, was converted to sound pressure level (SPL); the data was further processed using one-third octave analysis and subsequent A-weighting as is customary in the wind noise community.

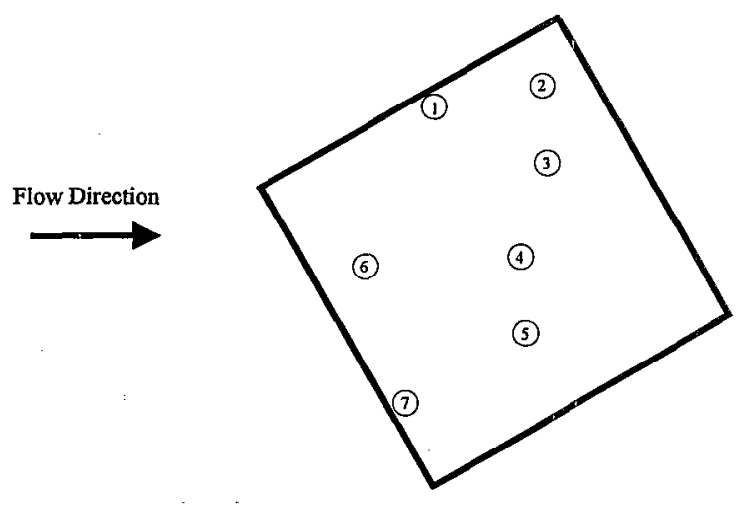

Figure 6. Surface pressure probe locations.

The results of the two cases for the cube at thirty degrees yaw are presented in Figs. 7 and 8 . Comparison of the two spectra show an increased spread among the probes with increased Reynolds number. The difference between the highest and lowest probe SPL is approximately $9 \mathrm{~dB}$. The positions of the probes relative to the averaged flow field structures can be determined by using Figs. 4 and 7 . The loudest probe, probe 3 , is located in the recirculation zone. Since true vortices do not form behind the leading edges, the most unsteady region on the top face of the cube would be this recirculation region. The next loudest probe, probe 2 , is at the edge of the same flow region. Probes 4 and 5 comprise the intermediate range with probes 1 , 6 , and 7 being the quietest. It should be noted that the threc quietcst probes lic very close to the cdges over which the oncoming flow turns. Although the velocity magnitude in these regions is quite high, the 
flow is still angles upward from the body as it recovers from turning the corner; the low noise level indicate that the flow surrounding these regions remains relatively constant. The region where probes 4 and 5 are located is where the flow 'reattaches' or becomes parallel to the body surface after making the sharp turn around the corner; it is not a true reattachment zone since a vortex bubble does not form.

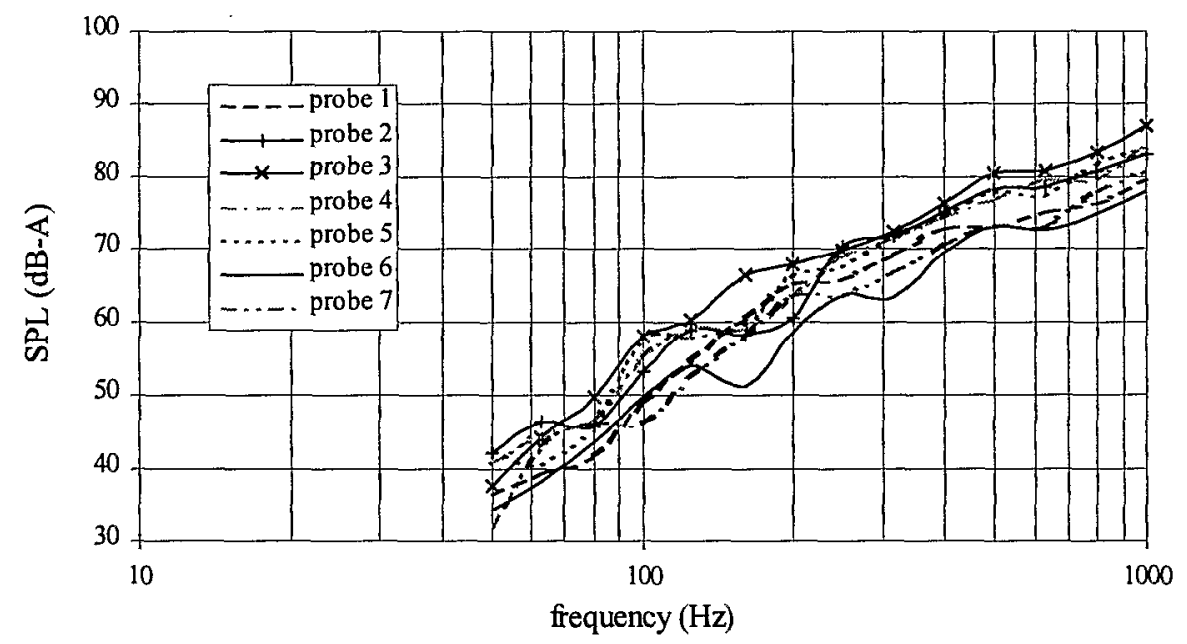

Figure 7. Pressure spectrum for $\mathrm{Re}=500$.

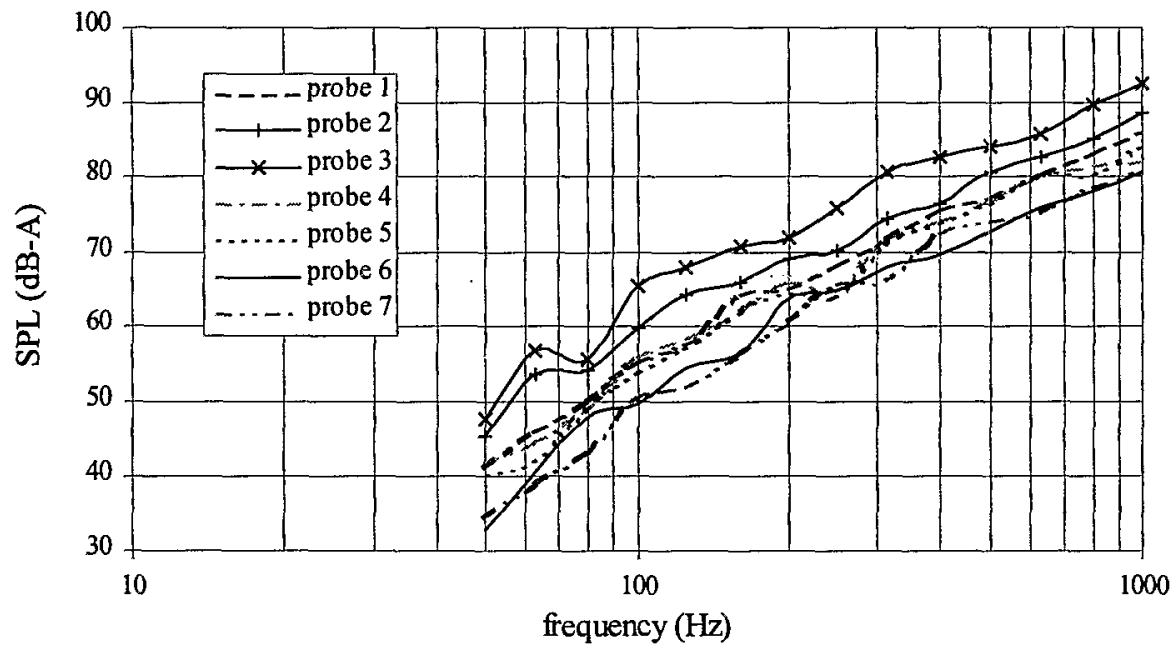

Figure 8. Pressure spectrum for $R e=1500$.

The pressure shown in Fig. 8 has a larger spread among the probes with the difference between the highest and lowest SPL typically being $12 \mathrm{~dB}$ across the entire frequency range. As with the $\mathrm{Re}=500$ case, probe 3 is the loudest probe. The increase in Reynolds number from 500 to 1500 causes the SPL of probe 3 to be 5-6 dB louder. Probe 3 sits quite close to the line where opposing flows meet, at the tail of the recirculation zone shown in Fig. 5. Again, the next loudest probe is probe 2 ; in this case, however, it is nearly $3.5 \mathrm{~dB}$ lower than probe 3 . In comparison to the flow field for $\mathrm{Re}=500$, Probe 2 lies within a 'reattaching' region; hence, we would expect the SPL to be lower for probe 2 than probe 3 based on the increased unsteadiness in the flow field in the vicinity of probe 3 . Probes 1,4 , and 5 comprise the intermediate range with probes 6 and 7 being the quietest. Along the edge where probe 1 lies, the flow is not overshooting the edge as much in the higher Reynolds number case is in the lower Reynolds number case, but rather is much more aligned with the cube surface than the flow at the location in the 
lower Reynolds number simulations. This is due to the position of the main recirculation zone, which is located farther upstream in the $\mathrm{Re}=1500$ case. The higher speed flow is subsequently directed around this region, resulting in amore parallel flow in the region of probe 1 . Thus the flow is more similar to that in the 'reattaching' region where probes 4 and 5 are positioned. The flow at the front edge still takes the corner at a rather high relative speed, overshooting the downstream portion of the front edge near the position of probes 6 and 7 .

\section{CONCLUSIONS}

-The two new methods developed to compute surface pressure at arbitrary points in the flow field in conjunction with vortex methods show substantial computational benefits over application of a full Poisson solver to the flow field. For simulations on a scale of these benchmark cases, the computation of the unsteady pressure at 25 arbitrary locations would take only $1-2 \%$ of the number of floating point operations necessary to compute the entire pressure filed using a direct Poisson solver. This is a very significant savings since the computations demands of a Poisson solver quickly surpass those of the VBEM when even a fairly low resolution volume grid is used. The capabilities of the new pressure methods were demonstrated in terms of both timeaveraged surface pressure coefficients-and unsteady surface pressure data which was then postprocessed to give spectral information.

This work is a successful first step in demonstrating the potential of the VBEM for use in computing unsteady flows to predict the pressure spectrum associated with the unsteady surface pressure fluctuations. The results of the calculations at Reynolds numbers of 500 and 1500 show the overall sound pressure level to increase significantly with Reynolds number, as expected. The VBEM coupled with a pressure method was also able to capture the different levels of pressure fluctuations associated with different flow regions.

\section{ACKNOWLEDGEMENTS}

The authors would like to thank Ahmed Ghoniem and Adrin Gharakhani for their assistance in using the VBEM code and for their helpful discussions regarding the various pressure methods.

\section{REFERENCES}

1. Najm, H., "Numerical Investigation of the Instability of Premixed Dump Combustors ", Ph.D. Thesis, MIT, Cambridge, MA, 1989.

2. Najm, H.N. and Ghoniem, A.F., "Convective Instability in a Dump Combustor", AIAA Journal., Vol. 29, 1991, pp. 911-919.

3. Gharakhani, A., "A 3-D Vortex-Boundary Element Method for the Simulation of Unsteady, High Reynolds Number Flows", Ph.D. Thesis, MTT, Cambridge, MA, 1995.

4. Ghoniem, A.F. and Sherman, F.S., "Grid-free simulation of "diffusion using random walk methods", J. Comp. Phys., Vol. 61, 1985, pp. 137.

5. Nakanishi, Y. and Kamemoto, K., "Procedure to Estimate Unsteady Pressure Distribution for Vortex Method", Trans. Japan Soc. Mech. Eng. 93-0537, 1993.

6. Lewis, R.I., Vortex Element Methods for Fluid Dynamic Analysis of Engineering Systems, (Cambridge University Press, Cambridge), 1991.

7. Babu, V. and Korpela, S.A., "On the Direct Solution of Poisson's Equation on a Non-uniform Grid", J. Comp. Phys., Vol, 104, 1993, pp. 9398.

8. Stapleford, W.R. and Carr, G.W., "Aerodynamic Noise in Road Vehicles, Part 1: The Relationship Between Aerodynamic Noise and the Nature of the Airflow", MIRA Report No. 1971/2, 1971.

9. Hucho, W.H., Aerodynamics of Road Vehicles: From Fluid Mechanics to Vehicle Engineering (Butterworth \& Co. Ltd., London), 1987. 